\title{
Heartmate II left ventricular assist device extensive driveline fracture: hanging on by a thread
}

\begin{abstract}
Sarah Schettle ${ }^{1 *}$, Richard Rodeheffer ${ }^{2}$, David Barbara ${ }^{3}$ and John Stulak ${ }^{1}$
${ }^{1}$ Mayo Clinic Department of Cardiovascular Surgery, USA

${ }^{2}$ Mayo Clinic Department of Cardiovascular Disease, USA

${ }^{3}$ Mayo Clinic Department of Anaesthesiology, USA

\section{Introduction}

Left ventricular assist device (LVAD) driveline damage is a postLVAD complication with limited nonsurgical treatment options; potentially necessitating LVAD exchange for severe complications. Case reports and small series studies have described driveline repair after fracture [1-4]. We describe the presentation and repair of an unusual, extensive driveline fracture and stabilization prior to surgical repair.
\end{abstract}

\section{Case report}

A 26-year-old female with dilated cardiomyopathy underwent HeartMate II LVAD implantation as destination therapy, eventually required device exchange on November 29, 2012 due to infection, and was supported with an EPC controller. Patient contacted the implanting centre reporting red heart alarms with associated chest pain and unusual LVAD sensations. After air transportation to the implanting centre and connection to the display monitor, speed drops from 9400 RPM to 0 RPM were noted with associated red heart alarms, chest pain, and hemodynamic decompensation. LVAD driveline was completely exposed, silicon connection was compromised, and plastic wire covering was blackened from wear and exposure over weeks (Figure 1). A controller exchange to a new pocket controller did not bring resolution to these events. The driveline was manipulated, pinched together, and pressed against the insertion section at the controller to minimize the frequency of pump stopping. Central access was established should inotrope support be required, imaging studies were obtained, and communication with Abbott was undertaken regarding potential external driveline repair. X-ray of the driveline revealed significant damage with only 1 of 6 wires intact (Figure 2). The patient proceeded with emergent surgical exchange to replace the damaged driveline which was uneventful and was extubated the same day. The patient continued to require manual stabilization of the driveline to maintain electrical contact and prevent pump stoppage during OR preparation and surgical dissection prior to device exchange.

\section{Discussion}

Driveline damage can occur with repeated wear over time. Driveline compromise prompts specific alarms with newer HeartMate pocket controllers. This patient was supported with the older EPC controller and did not have access to a display module or backup controller at the time of the events described; thus, red heart alarms that transpired left a broader differential of potential causes. Patients supported with EPC controllers with suspected driveline fracture should be maintained on battery support as outlet power can prompt a short to ground and pump

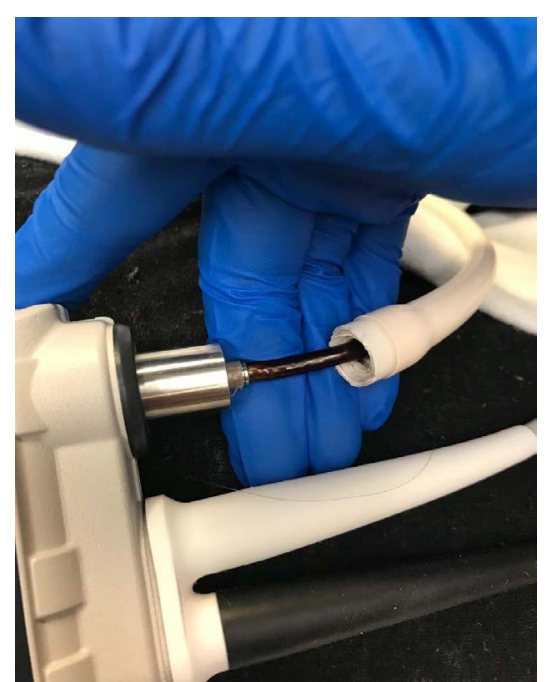

Figure 1. LVAD driveline reflecting silicone damage, discontinuity, and environmental exposure

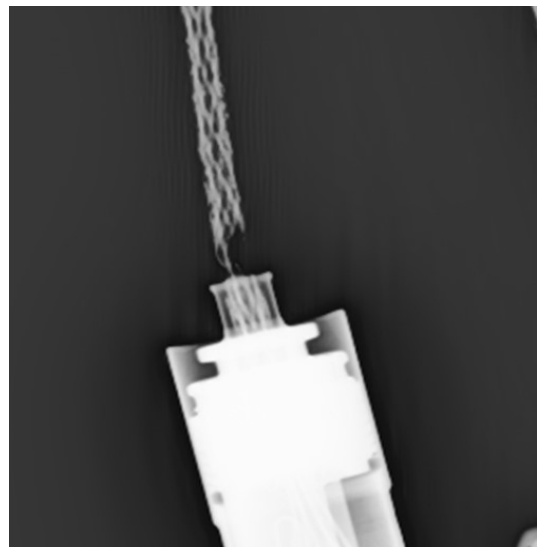

Figure 2. X-ray image of LVAD driveline revealing significant damage with only 1 of 6 wires intact

${ }^{\star}$ Correspondence to: Sarah Schettle, PA-C, MS, Mayo Clinic Department of Cardiovascular Surgery, USA, E-mail: schettle.sarah@mayo.edu

Key words: cardiomyopathy, cardiovascular surgery, treatment, mortality/ survival, ventricular assist device

Received: August 28, 2018; Accepted: September 05, 2018; Published: September 07, 2018 
stoppage. This patient's driveline had silicone damage and discontinuity for some time as the clear covering around the driveline wires had become dark brown with environmental exposure (Figure 1). Extent and location of driveline compromise can be difficult to evaluate with $\mathrm{x}$-ray images; however, this patient's images were unique in the clear demonstration of fractures. Generally, external splicing repair of the driveline is attempted before surgical repair if only one of the two paired wires in a driveline is suspected to be damaged. If driveline damage is extensive, thought to be internal, or if hemodynamic compromise is associated, more urgent operative intervention for exchange of the LVAD to replace the driveline needs to be considered. For this patient, manipulation of the driveline even a few millimetres resulted in pump stoppage and hemodynamic collapse; the extent of presentation in this case was dramatic and required constant human stabilization during all the time preceding definitive exchange. In some situations where patients are deemed non-operative, an ungrounded cable may be provided. Early identification of suspected LVAD driveline fracture is necessary to allow for patient stabilization, verification and ascertaining extent of compromise and evaluation of appropriate interventions to sustain ongoing LVAD support.

\section{Sources of Funding sources, Disclosures}

None of the authors has a financial relationship with a commercial entity that has an interest in the subject of the presented manuscript or other conflicts of interest to disclose.

\section{Acknowledgements}

We would like to acknowledge Jessica Miller, RN for her care of this patient.

\section{References}

1. Stulak JM, Schettle S, Haglund N (2017) Percutaneous Driveline Fracture After Implantation of the HeartMate II Left Ventricular Assist Device: How Durable is Driveline Repair? ASAIO journal 63: 542-545. [Crossref]

2. Boyechko Y, Tribble T, Guglin M (2016) Fatal Flaw - Driveline Fracture as a Rare but Serious Complication of Mechanical Circulatory Support with Left Ventricular Assist Devices. The Journal of Heart and Lung Transplantation 35: S265-S266.

3. Schima H, Stoiber M, Schloglhofer T, Hartner Z, Haberl T, et al. (2014) Repair of left ventricular assist device driveline damage directly at the transcutaneous exit site. Artificial organs 38: 422-425. [Crossref]

4. Kormos RL, McCall M, Althouse A (2017) Left ventricular assist device malfunctions: It is more than just the pump. Circulation 136: 1714-1725. [Crossref]

Copyright: (C2018 Schettle S. This is an open-access article distributed under the terms of the Creative Commons Attribution License, which permits unrestricted use, distribution, and reproduction in any medium, provided the original author and source are credited. 